\title{
Use of Polarized Light in the Diagnosis of "Fuzz" Granuloma in the Conjunctiva of a Child
}

\author{
Andrew R. Dorion ${ }^{a} \quad$ Gordon Crabtree $^{b} \quad$ R. Patrick Dorion ${ }^{c}$ \\ aSydney Kimmel School of Medicine at Thomas Jefferson University, Philadelphia, PA, USA; \\ ${ }^{b}$ Department of Ophthalmology, Geisinger Medical Center, Danville, PA, USA; 'Department \\ of Laboratory Medicine, Geisinger Medical Center, Danville, PA, USA
}

\section{Keywords}

Fuzz Ball granuloma Synthetic fiber granuloma Polarized light microscopy

\begin{abstract}
Synthetic fiber granulomas, also known as "Teddy Bear" granulomas or "Fuzz Ball granulomas," are usually seen in childhood. We present a case of a 5-year-old child with a "Fuzz Ball" granuloma of the conjunctiva. The resected specimen was processed routinely. The use of polarized light during microscopic examination of the resected tissue made the diagnosis evident due to the prominent birefringence of the synthetic foreign material fibers.
\end{abstract}

\section{Introduction}

Synthetic fiber granulomas have been reported in the literature as "Teddy Bear" granulomas or "Fuzz Ball" granulomas [1-9]. This entity usually presents unilaterally in the inferior fornix of children. Blankets, fabric toys (teddy bears for instance), and clothing in proximity to the eye are believed to cause inoculation of the fibers into the conjunctiva. Given time, the body reacts to the foreign material eliciting a granulomatous response. Synthetic fiber granulomas are uncommon but thankfully are easily removed by simple excision, without major surgery. During microscopic examination of resected specimens, the use of polarized light considerably enhances the appearance of synthetic foreign material. We present a case of a "Fuzz Ball" granuloma in a child where the diagnosis was facilitated by a clinical suspicion and polarizing microscopy of the resected specimen. 
Dorion et al.: Diagnosing Fuzz Granuloma with Polarized Light

\section{Case Report}

A 5-year-old male presented to an acute care facility with a foreign body sensation of the left eye for several days. The patient had been given a stuffed cat toy, which he had been sleeping with for about 2-3 weeks (Fig. 1). The physician noticed a papule in the left lower eyelid and attempted to remove it but was not able to do so. Thus, the patient was referred to the ophthalmology clinic of a tertiary care facility for further management. Signed informed consent to report this case was obtained from this patient's mother.

Evaluation of the patient's left conjunctiva revealed $1+$ injection, and in the lower, fornix was a $0.3 \times 0.2 \mathrm{~cm}$ fleshy pink lesion with a single eyelash attached. This was removed in its entirety with forceps and sent to the pathology laboratory for routine processing.

The anatomic pathology laboratory received a tan soft piece of tissue measuring 0.2 $\times 0.2 \times 0.1 \mathrm{~cm}$. The specimen was entirely submitted and processed for hematoxylin and eosin (H\&E) staining. Microscopic examination revealed a portion of conjunctiva with a prominent chronic inflammatory infiltrate and a few neutrophils. Also noted was a granulomatous response to synthetic fibers (Fig. 2). With polarized light, the synthetic fibers were prominently birefringent (Fig. 3). After removal of the lesion, the patient was treated with topical tobramycin-dexamethasone drops, and the recovery was uneventful. Unfortunately, no gross photograph is available of the lesion in situ. At the time of removal, it was decided not to take a photograph due to the patient's increased anxiety during the procedure.

Fig. 1. Cat toy.

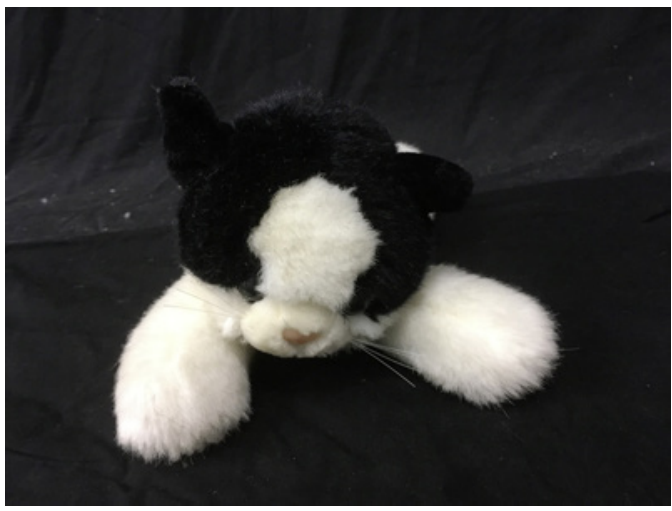

Fig. 2. Granulomatous response to synthetic fibers.

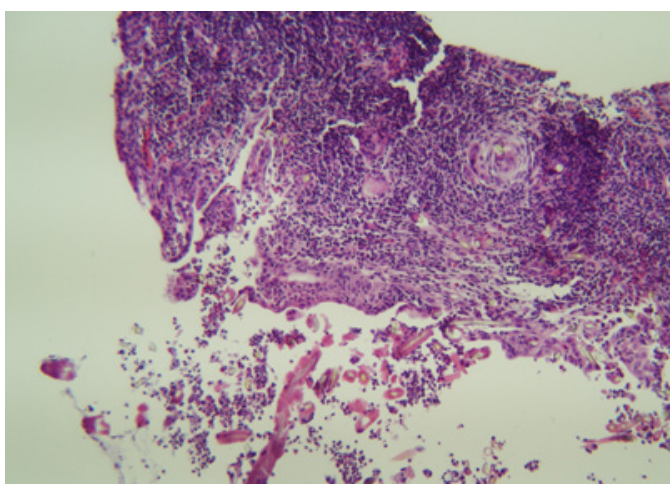


Fig. 3. Synthetic fibers with polarized light.

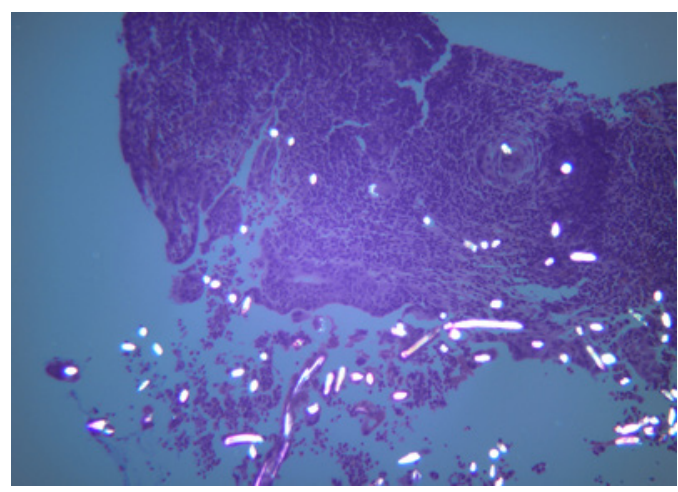

\section{Discussion/Conclusion}

The diagnosis of a synthetic fiber granuloma depends on a clinical suspicion and microscopic examination of the resected specimen. Routine H\&E processing is sufficient for an adequate assessment of the sample. During examination of the H\&E slides, the granulomatous process is usually evident; however, the synthetic fibers are not readily apparent since they do not stain with routine H\&E. The same microscopic slide when examined with a polarizing microscope makes the highly birefringent fibers very prominent thus facilitating the diagnosis of a "Fuzz Ball" granuloma. Being aware of such entity can facilitate the diagnosis for ophthalmologists and pathologists alike.

\section{Acknowledgement}

We gratefully acknowledge Shannon L. Sheikh for her assistance in the submission of this manuscript.

\section{Statement of Ethics}

Signed informed consent to report this case was obtained from this patient's mother.

\section{Conflict of Interest Statement}

The authors have no conflicts of interest to declare.

\section{Funding Sources}

No funding was received in relation to the preparation of this report.

\section{Author Contributions}

Andrew R. Dorion: drafting of the manuscript and literature review. Gordon Crabtree: patient assessment a treatment and manuscript review. R. Patrick Dorion: pathologic examination of tissue, obtaining photographs of the case, and manuscript review. 


\section{References}

1 Aliakbar-Navahi R, Roozitalab MH, Ashraf MJ, Hakimzadeh A. Synthetic fiber "Teddy Bear" conjunctival granuloma; a case report. J Ophthalmic Vis Res. 2015 Jul-Sep;10(3):342-4.

2 Mak ST, Lui YH, Li KK. Synthetic fibre granuloma of the conjunctiva. Hong Kong Med J. 2015 Feb;21(1):77-9.

3 Farooq MK, Prause JU, Heegaard S. Synthetic fiber from teddy bear causing keratitis and conjunctival granuloma: a case report. BMC Ophthalmol. 2011 Jun 20;11:17.

4 Schmack I, Kang SJ, Grossniklaus HE, Lambert SR. Conjunctival granulomas caused by synthetic fibers: report of two cases and review of literature. J AAPOS. 2005 Dec;9(6):567-71.

5 Enzenauer RW, Speers WC. Teddy bear granuloma of the conjunctiva. J Pediatr Ophthalmol Strabismus. 2002 Jan-Feb;39(1):46-8.

6 Yang YF, James CR. A conjunctival synthetic fibre granuloma in a child. Eye. 1996;10(Pt 1)(Pt-1):143-5.

7 Lueder GT, Matsumoto B, Smith ME. Pathological case of the month. Synthetic fiber granuloma ("Teddy Bear" granuloma). [Review]. Arch Pediatr Adolesc Med. 1996 Mar;150(3):327-8.

8 Lueder GT. Synthetic fiber granuloma. Arch Ophthalmol. 1995 Jul;113(7):848-9.

9 Ferry AP. Synthetic fiber granuloma. "Teddy Bear" granuloma of conjunctiva. Arch Ophthalmol. 1994 Oct; 112(10):1339-41. 